\title{
Dysembryoplastic neuroepithelial tumor (DNET) and focal cortical dysplasia: Case report of two pediatric patients with imaging features
}

\author{
Monwabisi Makola ${ }^{1}$, Kim M. Cecil*2 \\ ${ }^{1}$ University of Cincinnati, College of Medicine, Cincinnati, OH, USA \\ ${ }^{2}$ Imaging Research Center, Cincinnati Children's Hospital Medical Center and Departments of Radiology, Pediatrics, \\ Neuroscience and Environmental Health, University of Cincinnati College of Medicine, Cincinnati, OH, USA
}

Received: March 1, 2017

Accepted: April 20, 2017

Online Published: April 23, 2017

DOI: $10.5430 /$ ijdi.v4n2p31

URL: https://doi.org/10.5430/ijdi.v4n2p31

\begin{abstract}
We describe the clinical course and imaging features from two children diagnosed with focal cortical dysplasia and dysembryoplastic neuroepithelial tumor. These two children share similar pathology, however, this case report illustrates how their clinical presentation, management and imaging features differed with one treated primarily as a neoplasm, while the other as focal cortical dysplasia. These two patients illustrate the characteristics and challenges encountered with pediatric presentation of DNET and FCD. For patient 1, the clinical course, imaging and spectroscopic analyses reflected at early stage the ultimate pathological features of the lesion. In contrast, for patient 2, the early imaging features minimize what ultimately is predominately DNET.
\end{abstract}

Key Words: Focal cortical dysplasia, Dysembryoplastic neuroepithelial tumor, Pediatric brain tumor, Magnetic resonance imaging, Magnetic resonance spectroscopy

\section{INTRODUCTION}

Focal cortical dysplasia (FCD) is a congenital abnormality of cortical development. FCD is one of the most common causes of pharmacoresistant focal epilepsy and is frequently found in association with dysembryoplastic neuroepithelial tumors (DNETs). ${ }^{[1,2]}$ DNETs are found in close to $20 \%$ of patients who undergo surgery for the treatment of chronic epilepsy, making them the second most common surgically resectable epileptic tumors. ${ }^{[3]}$ Despite their prevalence in treatment resistant focal epilepsy patients, DNETs are rare tumors, accounting for close to $1 \%$ of primary CNS tumors. ${ }^{[3]}$ We describe two pediatric patients with DNET and FCD diagnoses producing dramatically distinct clinical courses: one presented primarily as a neoplasm, while the other presented as FCD. Imaging and pathological features are illustrated within a case report.

\section{Case PResentation}

\subsection{Patient 1}

The patient is a six-year old female with a history of chronic otitis media and accommodative esotropia. She initially presented with a four-week history of seizures. She exhibited mild appendicular hypotonia on neurological examination. Videotaped electroencephalogram (EEG) monitoring identified epileptiform discharges localized in the right temporal lobe with intermittent slowing in the right central head re-

\footnotetext{
*Correspondence: Kim M. Cecil, PhD; Email: kim.cecil@cchmc.org; Address: Cincinnati Children’s Hospital Medical Center, Imaging Research Center, MLC 5033, 3333 Burnet Avenue, Cincinnati, OH 45229, USA.
} 
gion. Seizure activity was characterized as complex partial seizures. A brain magnetic resonance imaging (MRI) examination was performed before and after administration of intravenous gadolinium contrast agent. A sharply defined lesion extended from the subcortical white matter of the superior right temporal lobe into the posterior aspect of the putamen, internal and external capsules and posterolat- eral thalamus. The lesion also bordered the atrium of the right lateral ventricle. The lesion demonstrated homogenous hyperintense signal in T2-weighted images, moderately heterogeneous hyperintense signal on fluid attenuated inversion recovery (FLAIR) images and slightly heterogeneous hypointense signal on T1-weighted images (see Figures 1-2).
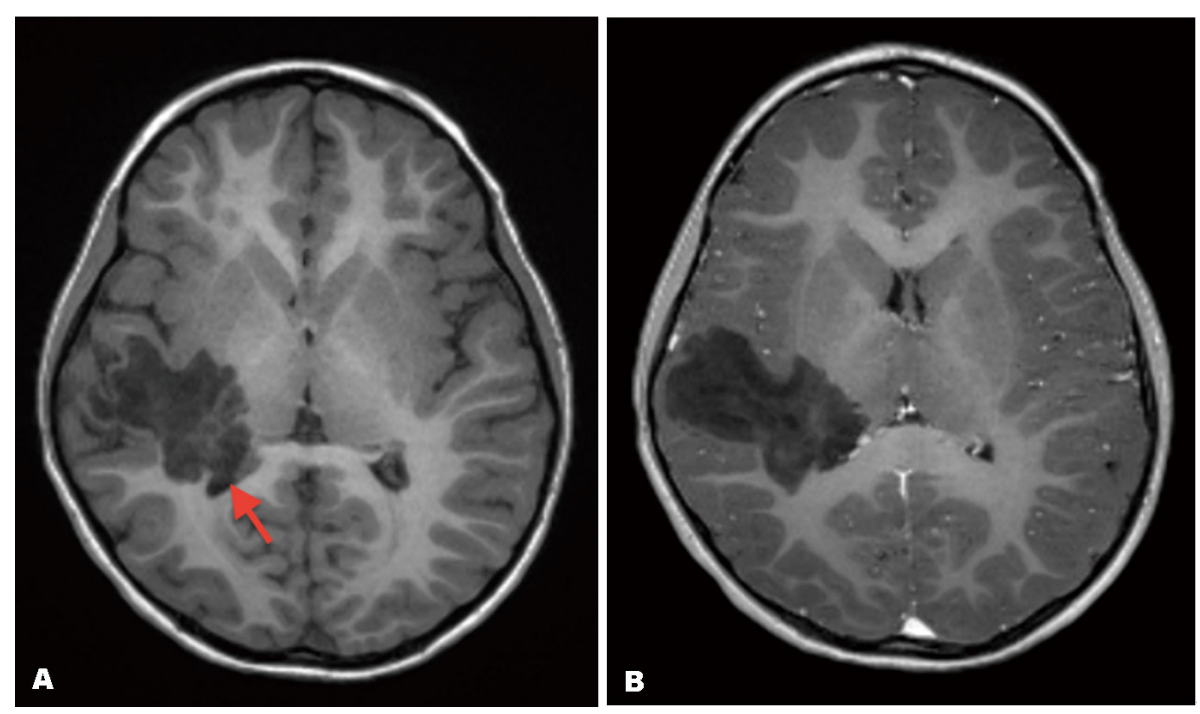

Figure 1. T1-weighted A) pre and B) post contrast T1-weighted images demonstrate hypointense signal defining the extent of the lesion without contrast enhancement in the right temporal lobe of a six-year old female (Patient 1). The lesion bordered the atrium of the right lateral ventricle (red arrow)
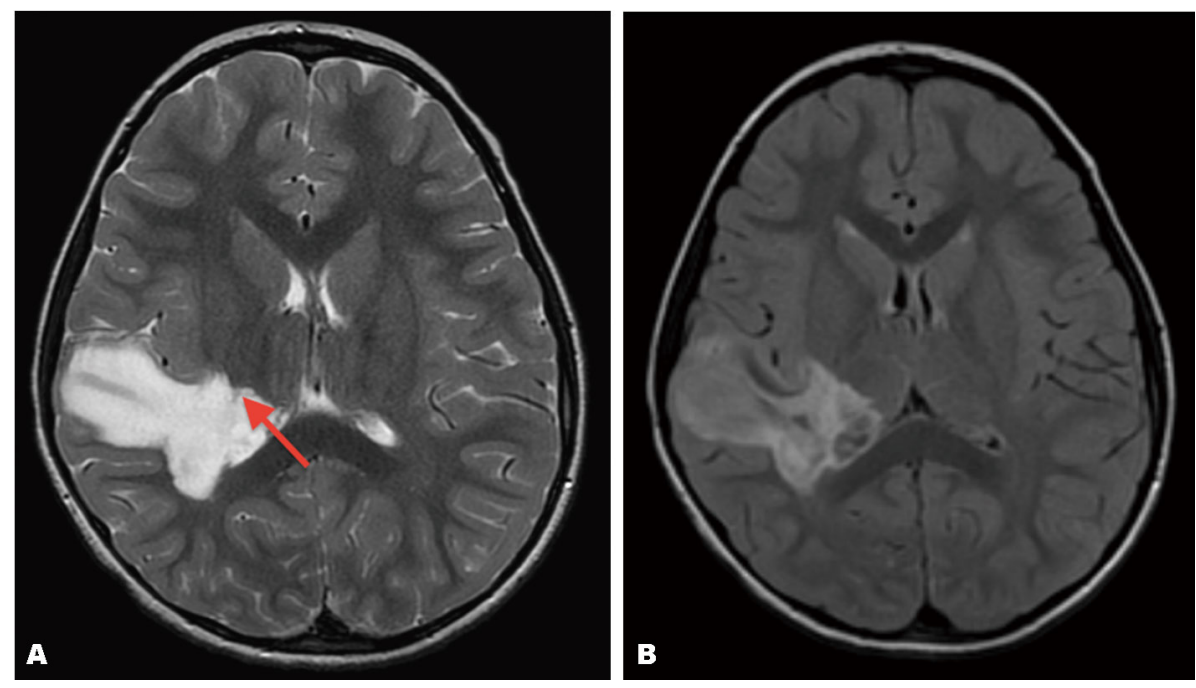

Figure 2. In a six-year old female (Patient 1), a sharply defined lesion extends from the subcortical white matter of the superior right temporal lobe into the posterior aspect of the putamen, internal and external capsules and posterolateral thalamus (red arrow). A: T2-weighted image demonstrates homogeneous hyperintense signal and B: FLAIR image shows heterogeneous hyperintense signal 
The lesion exerted no mass effect on surrounding structures. There was no evidence of diffusion restriction or contrast enhancement within the lesion. No evidence of abnormal signal intensity or abnormal vessels was seen on susceptibilty weighed images.
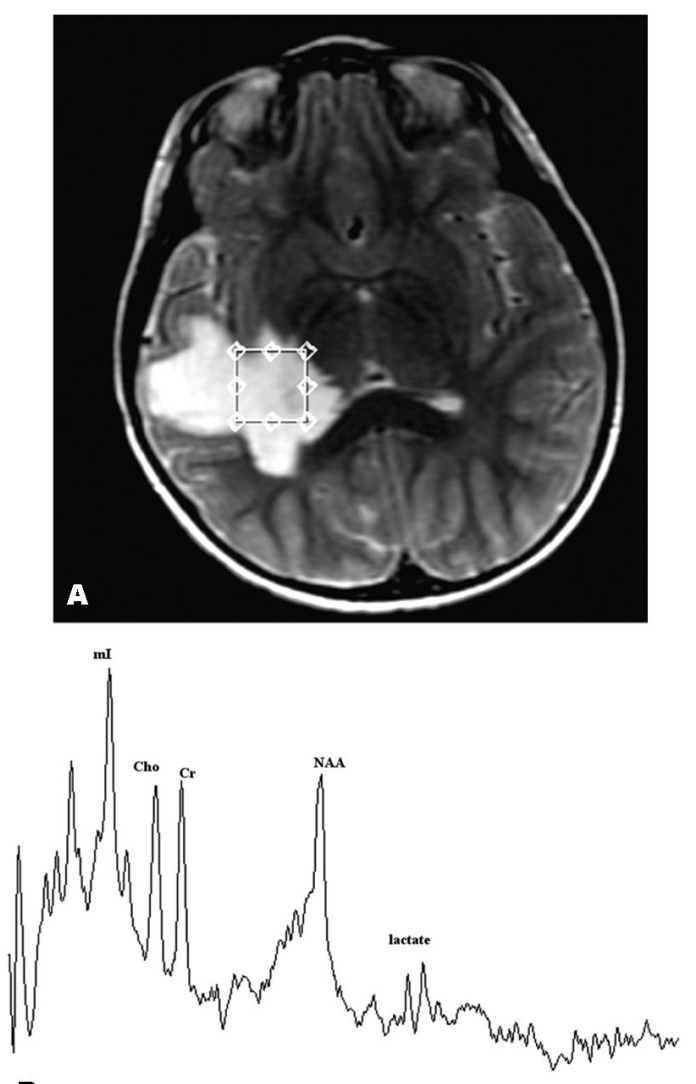

B
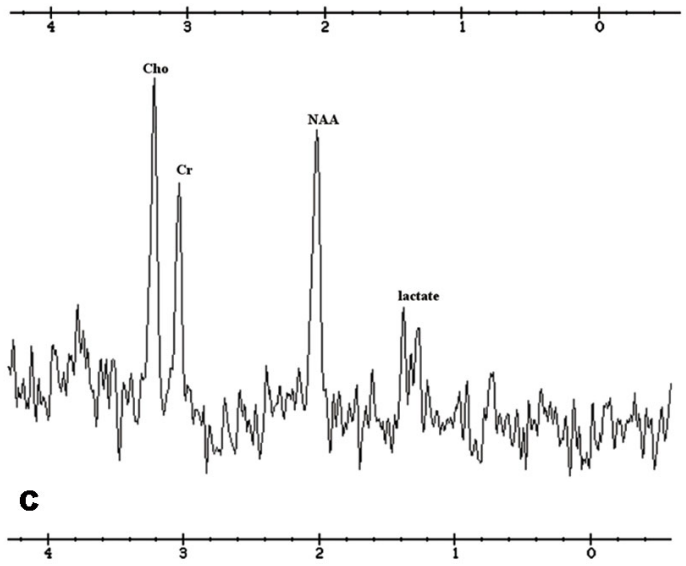

Figure 3. For a six-year old female (Patient 1). A) MRS voxel location (white box); B) Short Echo proton MRS and C) Long Echo proton MRS indicate elevated myo-inositol $(3.5 \mathrm{ppm})$ and lactate $(1.3 \mathrm{ppm})$ with diminished $\mathrm{N}$-acetyl aspartate (NAA, $2.0 \mathrm{ppm})$. Creatine $(\mathrm{Cr}, 3.0 \mathrm{ppm})$ and choline (Cho, $3.2 \mathrm{ppm}$ ) levels are within normal limits

Single voxel proton MR spectroscopy (MRS) was performed Published by Sciedu Press at 1.5 Tesla MRI field strength within the largest portion of the lesion, using short and long echo acquisitions (see Figure 3). Qualitatively, the spectrum demonstrated elevated lactate, myo-inositol and an unassigned resonance $3.8 \mathrm{ppm}$. The level of $\mathrm{N}$-acetyl aspartate (NAA) was reduced; creatine $(\mathrm{Cr})$ and choline (Cho) levels were within normal limits for age and location. Magnetoencephalography indicated localization adjacent to the lesion, however, there was peri-lesion irritability extending anterior, posterior and inferior from the lesion.

The patient underwent a right frontotemporal craniotomy with partial resection of the tumor as the risks of infarction or functional deficit from injury to deep brain structures outweighed the benefit of a gross total resection (see Figure 4). The child was seizure free following the surgery.
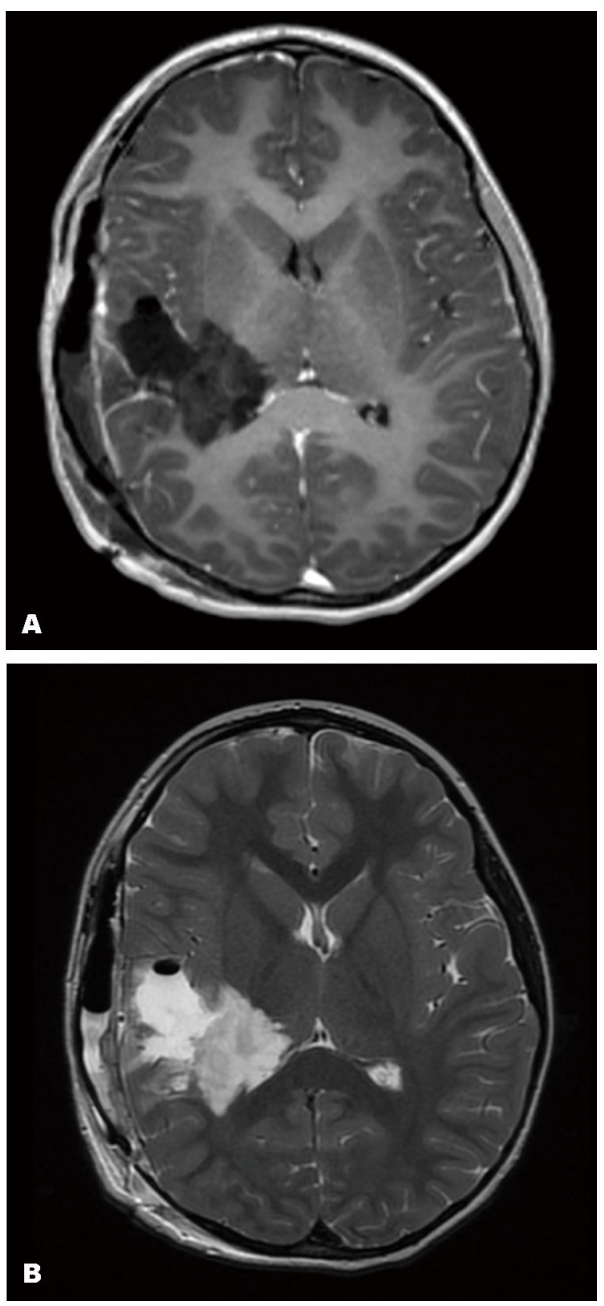

Figure 4. Post surgical A) T1 weighted images and B) T2 weighted images illustrate post-surgical changes with a partial resection of the lesion in a six year old female (Patient 1)

Upon pathological analysis, there was evidence of dyslam- 
ination within the cerebral cortical gray matter with focal microcolumn formation. One fragment had a collection of oligodendroglial-like cells within the underlying white matter and percolating into the gray matter. Several fragments showed a lightly myxoid background with embedded oligodendroglial cells with scattered normal appearing neurons floating within the myxoid material. CD34 highlights only vascular structures. MiB-1 is quite low with approximate $1 \%$ positivity. The pathology was consistent with a DNET and FCD type IIIb.

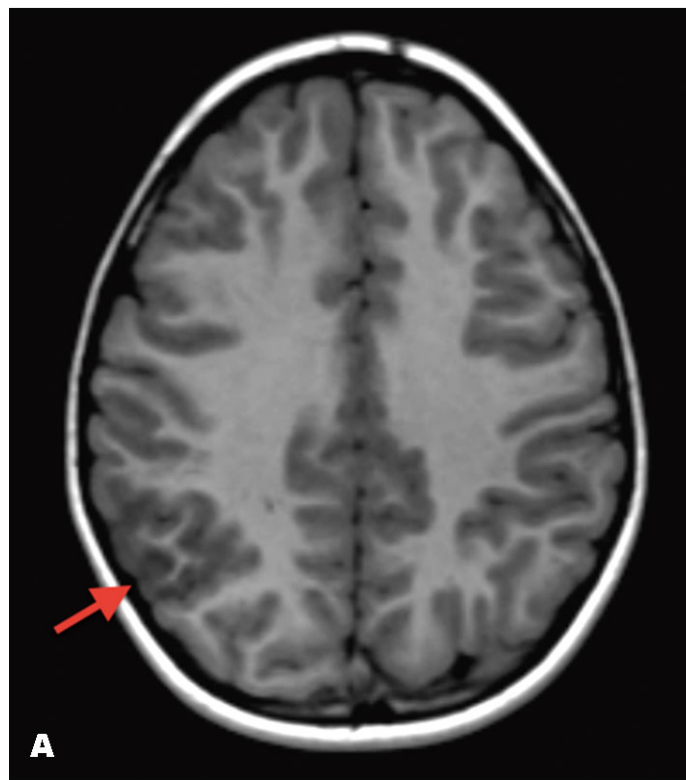

\subsection{Patient 2}

A male patient initially presented at 7 years of age with a history of episodes consistent for seizures including a maternal family history of seizures. Sleep deprived EEG was normal. A brain MRI performed before and after administration of intravenous gadolinium contrast agent revealed a wedge-shaped lesion within the right parietal cortex positioned posterior and inferior to the central sulcus. Ill-defined intermediate and hyperintense signal within the adjacent periatrial white matter was appreciated on T2-weighted and FLAIR images (see Figures 5-6).

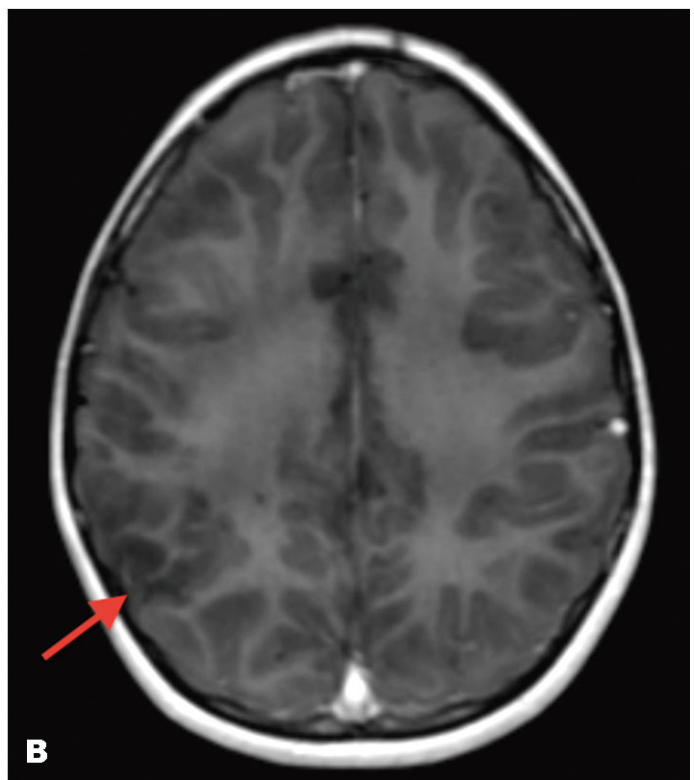

Figure 5. A seven-year old male (Patient 2) presents with a wedge-shaped lesion (red arrow) within the right parietal cortex positioned posterior and inferior to the central sulcus. A comparison of T1-weighted A) pre and B) post contrast imaging do not reveal gadolinium contrast uptake within the cortical lesion
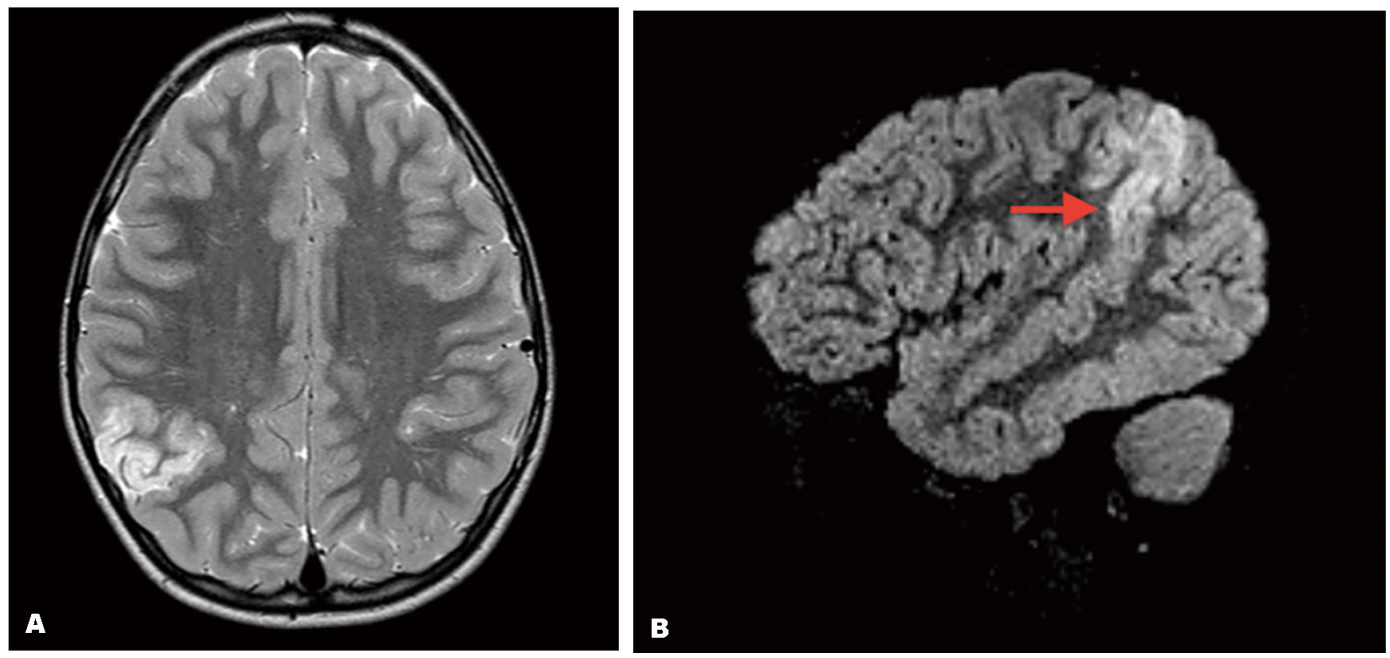

Figure 6. Baseline A) T2-weighted and B) FLAIR imaging illustrate hyperintense signal within the cortical, subcortical and adjacent periatrial white matter (red arrow) regions of the lesion for a seven-year old male 
There was no evidence of altered susceptibility, contrast enhancement nor diffusion restriction within the lesion. Single voxel proton MRS was performed at 1.5 Tesla MRI field strength within the largest portion of the lesion. The spectrum demonstrated with semi-quantitative analyses a slightly elevated myo-inositol level (see Figure 7).

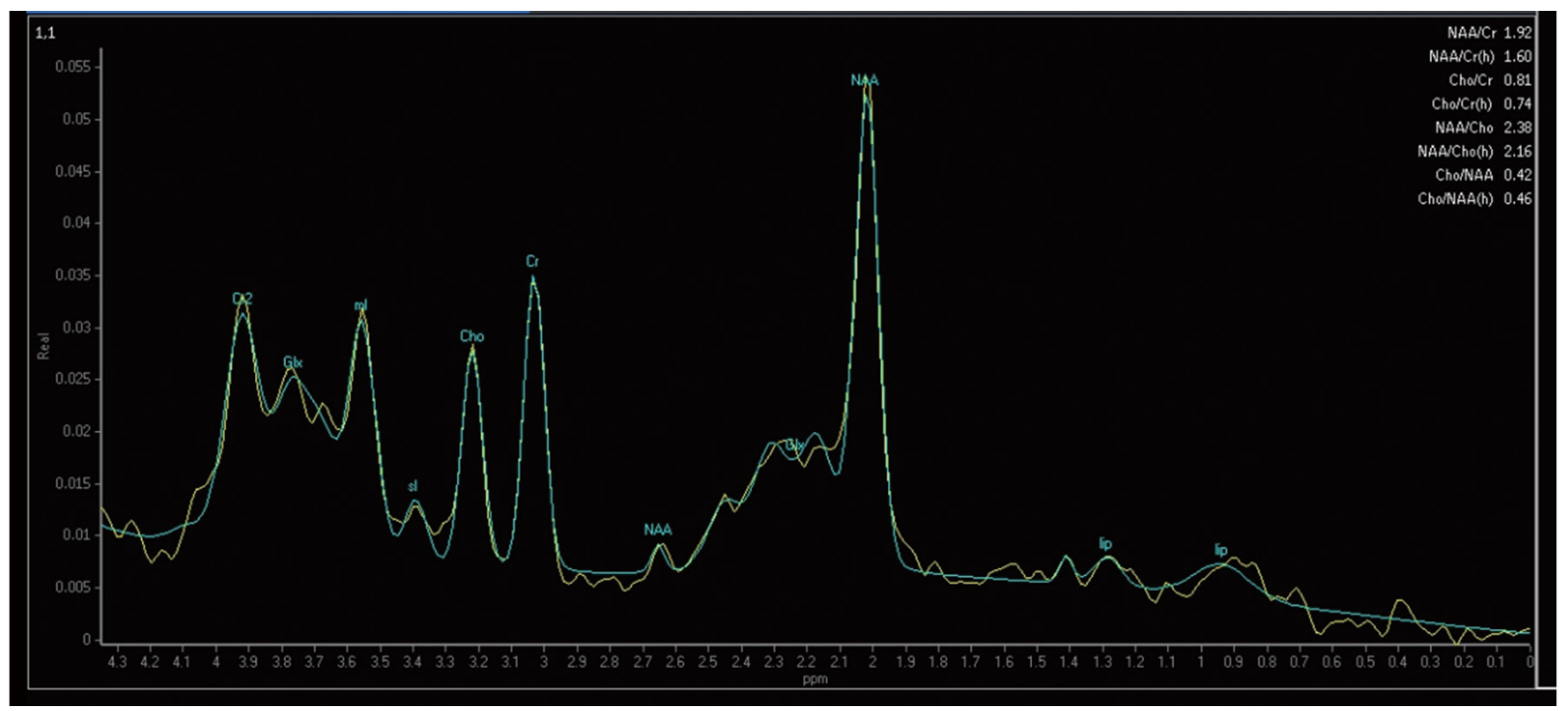

Figure 7. Baseline short echo proton MRS demonstrates near normal levels of $\mathrm{N}$-acetyl asparate (NAA), creatine (Cr), choline (Cho) with a slight elevation of myo-inositol (mI) at $3.5 \mathrm{ppm}$ for a seven-year old male

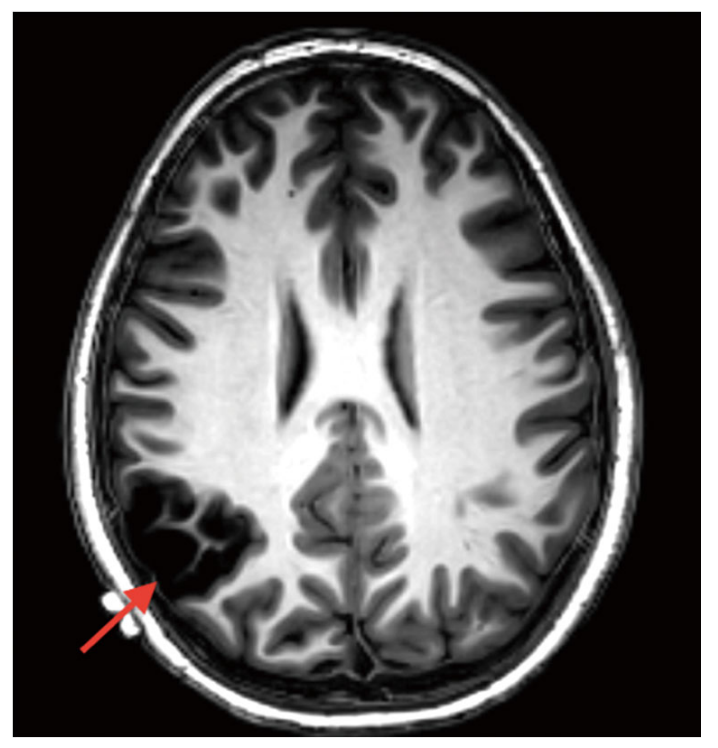

Figure 8. Imaging acquired before surgical resection for Patient 2, at 11 years of age, with Axial T1-weighted sequence demonstrating increased hypointensity of the cortical lesion (red arrow)

The levels of $\mathrm{N}$-acetyl aspartate, creatine and choline were within normal limits for age and location. There was no appearance of lactate. MRI examinations were stable for a year. The patient's focal seizures, which were secondarily generalized, were managed with antiepileptic medications. The patient exhibited behavioral problems, which were psychiatrically diagnosed and managed. The patient returned Published by Sciedu Press to imaging approximately two and a half years later. The signal characteristics intensified with increased $\mathrm{T} 2$ and decreased $\mathrm{T} 1$ signal within the cortical portion of the lesion (see Figures 8-9).

MRI demonstrated interval growth of the lesion. Subsequent videotaped EEG monitoring identified intermittent slowing within the bi-frontal head region. Intermittent high amplitude spikes and slow waves, with electroclinical seizures were recorded in the right frontal region. Seizure burden increased and were proven to be medically refractory despite treatment with multiple antiepileptic medications. The patient underwent a right parietal craniotomy for tumor resection. The patient seizures did not resolve following surgery. Post-surgical MRI identified several regions, which may represent postoperative changes or residual lesion (see Figure 10).

Upon pathological analysis, the lesion was classified as a DNET tumor with vaguely nodular, moderately cellular collection of cells with generally round regular nuclei, oligodendroglial-like with variable amounts of associate mucin. Scattered "floating neurons" were noted in the mucin rich areas. Occasional mitotic figures were identified without evidence of hypercellularity, vascular proliferation or necrosis. No Rosenthal fibers or eosinophilic granular bodies were detected. MiB-1 confirmed a generally low proliferation index ranging from $1 \%$ to $3 \%$. CD34 highlighted scattered foci of a single cell and mossy parenchymal positivity within several foci of the neoplasm. 

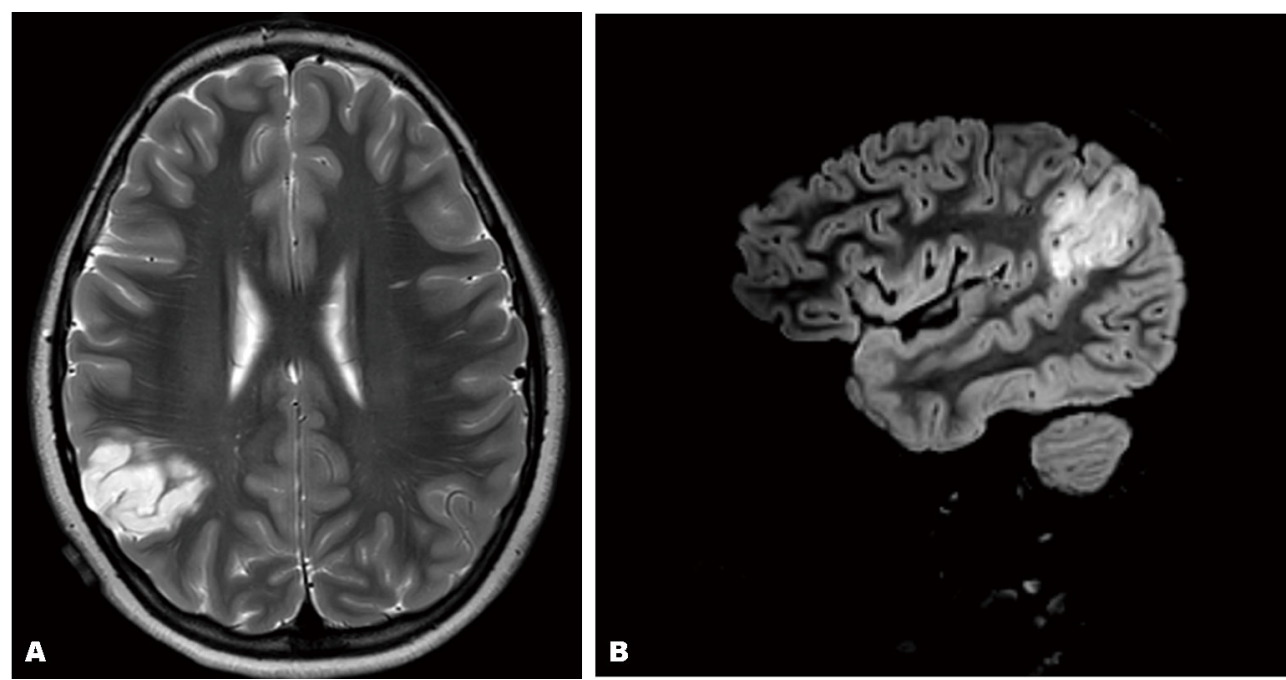

Figure 9. Patient 2, at 11 years of age, imaging indicates interval growth and increased hyperintensity associated with the lesion in the A) Axial T2-weighted and B) Sag 3D FLAIR images
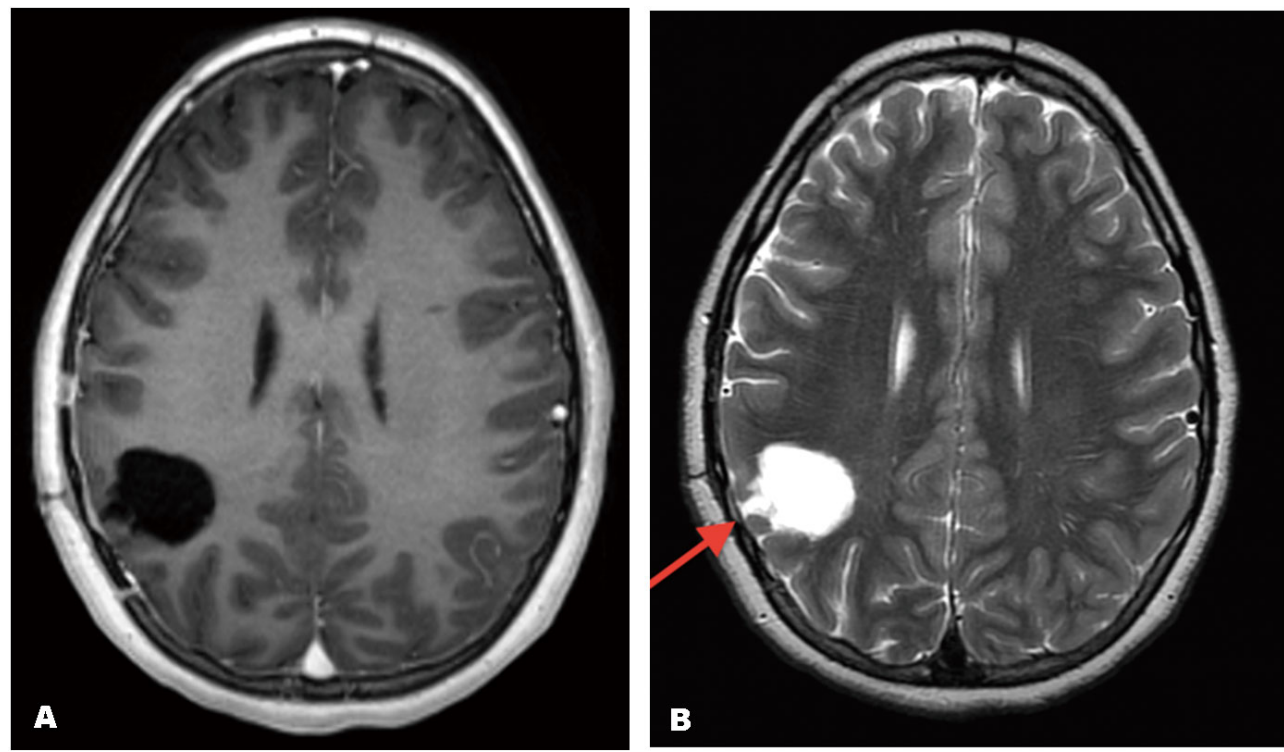

Figure 10. Post surgical A) T1-weighted images and B) T2-weighted images in an 11 year old male (Patient 2) identified post-surgical changes or residual lesion at the lateral edge (red arrow) of the resection cavity

\section{Discussion}

Long-term epilepsy associated-tumors (LEATs) correspond to low grade, slow growing, cortical based tumors demonstrating early-onset epilepsy refractory to anticonvulsant treatment. LEATs may be associated with cortical dysplasias. The predominant LEATs include ganglioglimoas and DNETs; however, other tumors can be classified in this grouping.

DNETs are benign, supratentorial, cortical lesions typically associated with intractable focal epilepsy in children and young adults. Patients rarely exhibit neurological signs beyond seizures and they generally have normal intelligence. ${ }^{[3]}$
MRI features of DNETs include swollen gyri and multilobulated/nodular lesions with a bubbly, cystic appearance. Scalloping of the skull where it meets the lesion is frequently seen, indicative of the slow growth of these tumors. ${ }^{[4]} \mathrm{Le}$ sions appear hypointense on $\mathrm{T} 1$ weighted imaging and hyperintense on T2 weighted and FLAIR imaging. Diffusion weighted imaging reveals high apparent diffusion coefficient while a lactate peak is sometimes seen on MRS. ${ }^{[4,5]}$ Pathological hallmarks of DNETs include the presence of glioneuronal elements comprising columns of oligodendrogliomalike cells and floating neurons. They frequently have a nodular structure and involve components resembling other tu- 
mors such as astrocytoma, oligodendroglioma, and oligoastrocytoma. ${ }^{[5,6]}$

FCD is a congenital abnormality of cortical development.
The hallmark feature of FCD is cortical dyslamination. FCD has several histological variations and is separated into three main types and 9 subtypes (see Table 1).

Table 1. Comparison of common DNET and FCD characteristics

\begin{tabular}{|c|c|c|}
\hline FEATURES & DNET & FCD \\
\hline Location & $\begin{array}{l}\text { Any cortical region } \\
\text { Temporal and frontal lobes most common }\end{array}$ & Any cortical region \\
\hline Appearance & $\begin{array}{l}\text { Swollen gyri } \\
\text { Bubbly, cystic appearance } \\
\text { Scalloping of the skull } \\
\text { Multilobulated/nodular }\end{array}$ & $\begin{array}{l}\text { Cortical thickening } \\
\text { Blurred grey-white matter junction } \\
\text { Segmental/lobar atrophy }\end{array}$ \\
\hline T1 Imaging & Hypointense lesion & Hypointense lesion \\
\hline T2 Imaging & Hyperintense lesion & $\begin{array}{l}\text { Hyperintense lesion and adjacent subcortical white } \\
\text { matter, often to ventricle }\end{array}$ \\
\hline FLAIR Imaging & Hyperintense rim, no edema & $\begin{array}{l}\text { Hyperintense lesion and adjacent subcortical white } \\
\text { matter, often to ventricle }\end{array}$ \\
\hline Diffusion Imaging & High apparent diffusion coefficient & $\begin{array}{l}\text { Decreased Fractional Anisotropy, increased } \\
\text { diffusivity outside of lesion }\end{array}$ \\
\hline MRS & May have elevated lactate peak & $\begin{array}{l}\text { Decreased N-acetyl aspartate (NAA)/Creatine and } \\
\text { NAA/Choline, elevated myo-inositol }\end{array}$ \\
\hline Pathology & $\begin{array}{l}\text { Simple: glioneuronal element (GNE) and floating cells } \\
\text { Complex: nodular/multinodular intracortical structure } \\
\text { Diffuse: diffuse cortical infiltration, sparse nodularity and GNE } \\
\text { Mixed: GNE and elements of other tumors }\end{array}$ & $\begin{array}{l}\text { Type 1: abnormal cortical lamination } \\
\text { Type 1a: radial lamination } \\
\text { Type 1b: tangential lamination } \\
\text { Type 1c: radial and tangential lamination } \\
\text { Type 2: dysmorphic neurons/balloon cells } \\
\text { Type 2a: dysmorphic neurons } \\
\text { Type 2b: dysmorphic neurons and balloon cells } \\
\text { Type 3: adjacent principal lesion } \\
\text { Type 3a: adjacent to hippocampal sclerosis } \\
\text { Type 3b: adjacent to glial or glioneuronal tumor } \\
\text { Type 3c: adjacent to vascular malformation } \\
\text { Type 3d: adjacent to lesion acquired early in life }\end{array}$ \\
\hline $\begin{array}{l}\text { Immuno- } \\
\text { histochemistry }\end{array}$ & $\begin{array}{l}\text { Commonly: CD34, calbindin, S-100 and OLIG-2 positive } \\
\text { Frequently: nestin and NeuN positive, 1p/19q co-deletions or 19q/PTEN loss } \\
\text { Rarely: Ki67 or MiB-1 positive }\end{array}$ & NeuN positive \\
\hline
\end{tabular}

MRI evaluation of FCD frequently reveals cortical thickening and blurring of the grey matter (GM)-white matter (WM) junction. Segmental or lobar hypoplasia and atrophy are also common findings. ${ }^{[2]}$ T1-weighted images display hypointense GM and WM while T2-weighted and FLAIR images reveal hyperintense GM and WM. The transmantle sign is commonly observed on T2-weighted and FLAIR images and features hyperintense signal stretching from a ventricle to the cortex. Diffusion tensor imaging reveals decreased fractional anisotropy and increased mean diffusivity in and around the lesion. ${ }^{[2]}$

Patient 1 illustrates these typical clinical, imaging, spectroscopic and pathological features for DNET. However, this patient also presented with features of FCD upon proton MR Spectroscopy. The striking elevation of myo-inositol strongly suggested FCD in the presence of normal creatine and choline with slightly diminished $\mathrm{N}$-acetyl aspartate levels. The levels of lactate detected on MRS supported diagno- sis of DNET. Pathological analysis confirmed the presence of FCD within resected regions of the lesion. The region sampled for proton MRS was not resected; this raises concerns for future recurrence of seizure activity in this patient.

Chan et al. evaluated the long-term seizure outcome following surgery for pediatric patients with a temporal lobe based DNET ${ }^{[7]}$ The mean duration of follow up was 10.8 years in their study. They reported that complete resection of DNET lesions produced a favorable postoperative seizure outcome without tumor recurrence or progression. The post-operative seizure outcome remained stable over the years; long-term seizure freedom could be predicted based on short-term follow up at 2 to 3 years. For cases with incomplete resection and persistent seizure activity, repeated surgery lead to improved seizure control. Chan et al. recommended avoiding adjuvant radiotherapy and chemotherapy in these patients.

The clinical management with antiepileptic medications, 
imaging and spectroscopy for Patient 2 favored the diagnosis of FCD for several years. Subsequent changes in seizure frequency and control combined with imaging appearance altered the clinical management towards surgical intervention. Despite a near complete surgical resection, seizure activity persists. Kasper and Kasper describe the urgent need to shorten disease duration prior to epilepsy surgery. ${ }^{[8]}$ Pelliccia et al. reported that pediatric patients are more responsive to epilepsy surgery than adults. ${ }^{[9]}$ Their analysis also found that temporal lobe localization, complete resection and shorter duration of epilepsy were the determinant factors for a favorable postoperative seizure outcome. Their findings also supported the early removal of LEATs to achieve better seizure freedom and improve cognitive development. Ramantani et al. argues that beyond seizure freedom, having shorter latency to surgery provides the opportunity to avert damage to the developing brain by seizures, and anti-epileptic drugs. ${ }^{[10]}$ The delay in accurate diagnosis of a LEAT for Patient 2 described in this study prognosticates for poor seizure control and an adverse overall developmental outcome.

\section{CONFlicts OF INTEREST Disclosure}

The authors have declared no conflicts of interest.

\section{REFERENCES}

[1] Blumcke I, Thom M, Aronica E, et al. The clinicopathologic spectrum of focal cortical dysplasias: a consensus classification proposed by an ad hoc Task Force of the ILAE Diagnostic Methods Commission. Epilepsia. 2011; 52(1): 158-174. PMid:21219302 https://doi.org/10.1111/j.1528-1167.2010.02777.x

[2] Widdess-Walsh P, Diehl B, Najm I. Neuroimaging of focal cortical dysplasia. J Neuroimaging. 2006; 16(3): 185-96. PMid:16808819 https://doi.org/10.1111/j.1552-6569.2006.00025.x

[3] Suh YL. Dysembryoplastic Neuroepithelial Tumors. J Pathol Trans] Med. 2015; 49(6): 438-49. PMid:26493957 https : //doi .org/10 $.4132 / j p t m .2015 .10 .05$

[4] Borja MJ, Plaza MJ, Altman N, et al. Conventional and advanced MRI features of pediatric intracranial tumors: supratentorial tumors AJR Am J Roentgenol. 2013; 200(5): W483-503. PMid:23617516 https://doi.org/10.2214/AJR.12.9724

[5] Thom M, Toma A, An S, et al. One hundred and one dysembryoplastic neuroepithelial tumors: an adult epilepsy series with immunohistochemical, molecular genetic, and clinical correlations and a review of the literature. J Neuropathol Exp Neurol. 2011; 70(10): 859-78. PMid:21937911 https://doi.org/10.1097/NEN.0b013e3182 302475
[6] Komori T, Arai N. Dysembryoplastic neuroepithelial tumor, a pure glial tumor? Immunohistochemical and morphometric studies. Neuropathology. 2013; 33(4): 459-68. PMid:23530928 https ://doi. org/10.1111/neup. 12033

[7] Chan CH, Bittar RG, Davis GA, et al. Long-term seizure outcome following surgery for dysembryoplastic neuroepthelial tumor. J. Neurosurg. 2006; 104(1): 62-9. PMid:16509148 https : //doi.org/10.3171/jns.2006.104.1.62

[8] Kasper BS, Kasper EM. New classification of epilepsy-related neoplasms: The clinical perspective. Epilepsy \& Behav. 2017; 67: 91-97. PMid:28110204 https://doi.org/10.1016/j.yebeh.2016.1 2.020

[9] Pelliccia V, Deleo F, Gozzo G, et al. Early and late epilepsy surgery in focal epilepsies associated with long-term epilepsy-associated tumors. J Neurosurg. Published online January 13, 2017. https : //doi.org/10.3171/2016.9. JNS161176

[10] Ramantani G, Stathi A, Brandt A, et al. Posterior cortex epilepsy surgery in childhood and adolescence: Predictors of long-term seizure outcome. Epilepsia. 2017; 58(3): 412-19. https ://doi .org/10.1 111/epi. 13654 